\title{
AN ORDERED FRAMEWORK FOR PARTIAL MULTIVALUED FUNCTORS
}

\author{
ALVEEN CHAND AND ITTAY WEISS
}

\begin{abstract}
The category Rel of sets and relations intimately ties the notions of function, partial multivalued function, and direct image under a function through the description of Rel as the Kleisli category of the covariant power set functor on Set. We present a suitable framework to obtain a similar relationship between the concepts of functor, partial multivalued functor, and the direct image under a functor.
\end{abstract}

\section{INTRODUCTION}

Partial multivalued functions arise naturally in the presence of uncertainty or partial information. In computer science, we mention [15] in the context of complexity theory and [11] in the context of multivalued relational databases, where partial multivalued functions appear prominently. In mathematics, multivalued functions are common-place in complex analysis and used in homotopy theory. Mathematically, a partial multivalued function $S \rightarrow T$ can be modeled as a relation from $S$ to $T$ or as a function $S \rightarrow \mathcal{P}(T)$ to the power set of $T$. Often, the sets $S$ and $T$ are endowed with extra structure, for instance an ordering, and the partial multivalued functions are to preserve that information in some sense. More generally, the domain $S$ and the codomain $T$ may be categories, and instead of a partial multivalued function one is interested in the notion of a partial multivalued functor. We now take two straightforward ad-hoc approaches to model the notion of a partial multivalued functor.

Extending the notion of a partial multivalued function to categories, we define a partial multivalued functor $F: \mathscr{C} \rightarrow \mathscr{D}$ between two small categories to consist of a partial multivalued function $F: \mathrm{ob} \mathscr{C} \rightarrow \mathrm{ob} \mathscr{D}$ and a partial multivalued function $F: \mathscr{C}\left(C, C^{\prime}\right) \rightarrow \bigcup_{D \in F C, D^{\prime} \in F C^{\prime}} \mathscr{D}\left(D, D^{\prime}\right)$, for all $C, C^{\prime} \in$ ob $\mathscr{C}$, such that the identity morphisms and composition are respected, in the sense that

- for all $C \in \mathrm{ob} \mathscr{C}$, if $d \in F\left(\operatorname{id}_{C}\right)$, then $d=\mathrm{id}_{D}$ for some $D \in F C$; and

- for all $c^{\prime \prime} \in \operatorname{mor} \mathscr{C}$ and $d^{\prime \prime} \in \operatorname{mor} \mathscr{D}$ with $d^{\prime \prime} \in F\left(c^{\prime \prime}\right)$, if $c^{\prime \prime}=c^{\prime} \circ c$ for some $c, c^{\prime} \in \operatorname{mor} \mathscr{C}$, then there exist $d \in F c$ and $d^{\prime} \in F c^{\prime}$ with $d^{\prime \prime}=d^{\prime} \circ d$.

Remark 1.1. Note that the naively more immediate condition for preservation of composition, namely that if $d \in F(c)$ and $d^{\prime} \in F\left(c^{\prime}\right)$, then $d^{\prime} \circ d \in F\left(c^{\prime} \circ c\right)$, is not a natural condition to impose. Indeed, the composability of $c^{\prime}$ with $c$ need not imply that of $d^{\prime}$ with $d$, and it seems rather contrived to demand that if $d^{\prime} \circ d$ happens to exist, then $d^{\prime} \circ d \in F\left(c^{\prime} \circ c\right)$.

With the obvious notions of identities and composition, all small categories and partial multivalued functors form the category Cat $\mathbf{~ p m v}_{\mathbf{p m}}$. Obviously, a functor is 
(by slight abuse of terminology) also a partial multivalued functor, and thus Cat is a subcategory $\mathbf{C a t}_{\text {pmv }}$.

The second approach is obtained by extending the notion of relation between sets to categories. A relation $R: \mathscr{C} \rightarrow \mathscr{D}$ between two small categories consists of a relation $R: \mathrm{ob} \mathscr{C} \rightarrow \mathrm{ob} \mathscr{D}$ and a relation $R: \mathscr{C}\left(C, C^{\prime}\right) \rightarrow \bigcup_{D R C, D^{\prime} R C^{\prime}} \mathscr{D}\left(D, D^{\prime}\right)$, for all $C, C^{\prime} \in \mathrm{ob} \mathscr{C}$, which are compatible with the identities and the compositions, in the sense that

- for all $C \in \mathrm{ob} \mathscr{C}$, if $\mathrm{id}_{C} R d$, then $d=\mathrm{id}_{D}$ for some $D \in \mathrm{ob} \mathscr{D}$ with $C R D$; and

- for all $c^{\prime \prime} \in \operatorname{mor} \mathscr{C}$ and $d^{\prime \prime} \in \operatorname{mor} \mathscr{D}$ with $c^{\prime \prime} R d^{\prime \prime}$, if $c^{\prime \prime}=c^{\prime} \circ c$ for some $c, c^{\prime} \in \operatorname{mor} \mathscr{C}$, then $d^{\prime \prime}=d^{\prime} \circ d$ for some $d, d^{\prime} \in \operatorname{mor} \mathscr{D}$ with $c R d, c^{\prime} R d^{\prime}$.

Remark 1.2. Analogously to Remark 1.1, the more naive demand that $R$ be a congruence for the composition, namely that if $c R d$ and $c^{\prime} R d^{\prime}$, then $\left(c^{\prime} \circ c\right) R\left(d^{\prime} \circ\right.$ $d$ ) suffers from the same ill-behaviour that the naive preservation of composition exhibits. We further note that the temptation of defining a relation from $\mathscr{C}$ to $\mathscr{D}$ to be a subcategory of $\mathscr{C} \times \mathscr{D}$ results in a notion that is hardly related to our notion of relation, as can easily be seen by direct inspection.

With the evident notions of identities and composition, all small categories and relations form the category $\mathbf{C a t}_{\text {rel }}$.

Theorem 1.3. The categories $\mathbf{C a t}_{\mathbf{p m v}}$ and $\mathbf{C a t}$ rel are isomorphic.

Proof. An isomorphism is given by the identity on objects, mapping a partial multivalued functor $F: \mathscr{C} \rightarrow \mathscr{D}$ to the relation $R$ where $C R D$ if $D \in F C$, and $c R d$ if $d \in F c$, respectively, for all objects and morphisms. The details are immediate.

The notions of function, direct image under a function, and partial multivalued function (i.e., a relation) are related in the following way. Consider the category Set of sets and functions and recall the covariant power set functor $\mathcal{P}$ : Set $\rightarrow$ Set, mapping a set to its power set and a function $f: S \rightarrow T$ to the direct image function $f_{\rightarrow}: \mathcal{P} S \rightarrow \mathcal{P} T$. This endofunctor is well-known to be part of a monad, known as the Manes monad, whose Kleisli category is Rel, the category of sets and relations. In light of the above construction, it is natural to expect the category $\mathbf{C a t}_{\mathbf{p m v}}$, and thus also its isomorphic copy $\mathbf{C a t} \mathbf{t}_{\text {rel }}$, to arise as the Kleisli category of a monad on Cat given on objects by some sort of power category construction, and on functors by a suitable direct image construction.

However, there is an immediate obstruction to such a result. It is well known that the naive notion of image of a functor need not be a subcategory of the codomain. A minimal example illustrating this is the functor $K$

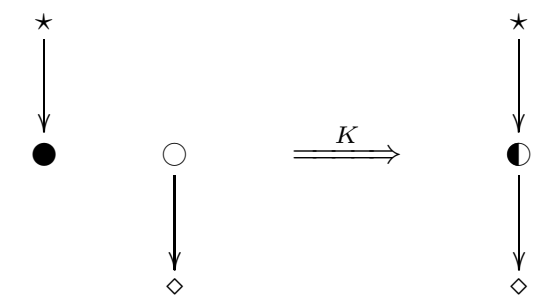

which is not expected to readily be reconciled with a covariant power category functor on Cat. 
The aim of this work is to present a category-based formalism extending the function/direct-image/partial-multivalued-function trio to a corresponding menage a trois of the concepts of functor, direct image under a functor, and partial multivalued functor. In more detail, denoting Set by $\mathbf{C a t}_{\mathbf{0}}$ and $\mathbf{R e l}$ by $\mathbf{R e l}_{\mathbf{0}}$, we construct suitable categories $\mathbf{C a t}_{\mathbf{1}}$ and $\mathbf{R e l}_{\mathbf{1}}$, whose objects are categories, and we obtain the diagram

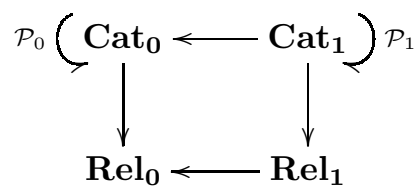

in which the top horizontal arrow is a morphism of monads, and the bottom part is the Kleisli construction of the top part. In fact, as a consequence of the obstruction mentioned above, this diagram expands to

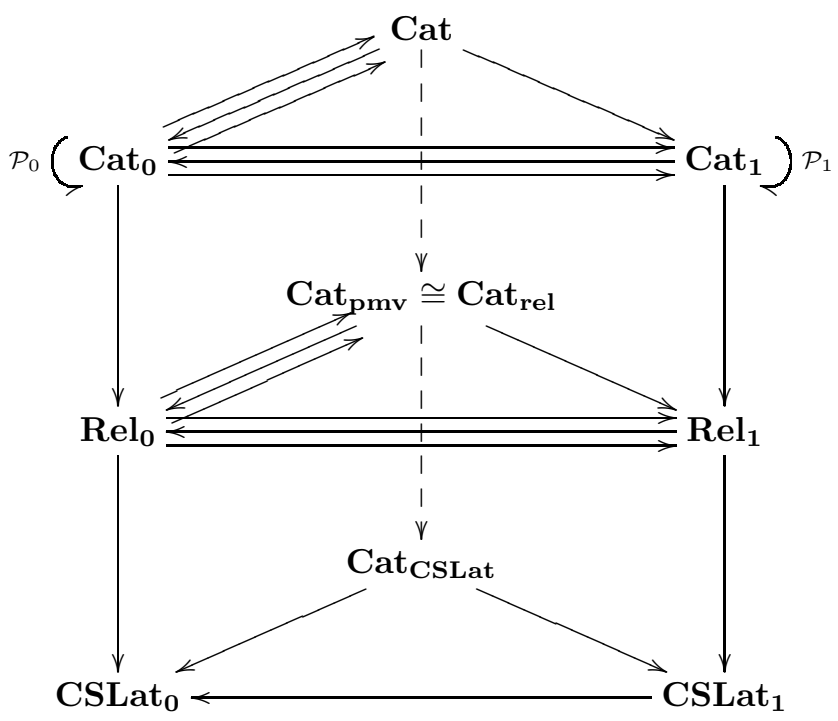

with $_{\mathbf{C a t}} \mathbf{p m v} \cong \mathbf{C a t}_{\text {rel }}$ appearing as a full subcategory of $\mathbf{R e l}_{\mathbf{1}}$. Since the EilenbergMoore category of $\mathcal{P}_{0}$ is $\mathbf{C S L a t}_{\mathbf{0}}$, the category of complete semi-lattices, we obtain at the bottom triangle extensions of it to categories.

In mathematics, the notion of partial multivalued functor appears in homotopy theory (see [8], 9]) where algebraic models are defined to be multivalued functors to Top on a category with a notion of homotopy. Multivalued functors are also discussed in [1], 3], and 10]. Quite generally, 2] discusses partial information, nondeterminacy, and multivaluedness in many contexts. Recently, stochastic relations are applied in such areas as computation, automata, and languages and programming (see e.g., [4, 5, 6]). The category of stochastic relations is the Kleisli category of the Giry monad ([7]), extending the Manes monad. The monad we present is a similar extension of the Manes monad not probabilistically but rather categorically.

The categorical prerequisites for reading this work are modest. We refer the reader to [12] for general information regarding categories, monads, the Kleisli construction, and the Eilenberg-Moore construction. 
The plan of the paper is as follows. Section 2 introduces categorders and functorders, which together form the extension $\mathbf{C a t}_{\mathbf{1}}$ of Cat for the required monad to act on. Section 3 and Section 4 describe, respectively, the power categorder of a categorder and the direct image functorder associated to a functorder, which together form the underlying functor $\mathcal{P}_{1}$ of the monad on $\mathbf{C a t}_{1}$. Section 5 then establishes the monad structure on $\mathcal{P}_{1}$, and Section 6 presents the main result, Theorem 6.1, obtaining the formalism of partial multivalued functors, as well as a brief investigation of the Eilenberg-Moore algebras of $\mathcal{P}_{1}$ as an extension of complete join semi lattices.

\section{Categorders And FunCtorders}

In this section we extend the category Cat of small categories and functors to the category Cat $_{1}$ which will serve as the ambient category for the power object monad we seek.

Definition 2.1. A category with order, or categorder, is a category $\mathscr{C}$ together with an ordering of each hom set $\mathscr{C}\left(C, C^{\prime}\right)$ such that for all morphisms $C \stackrel{c_{1}, c_{2}}{\longrightarrow} C^{\prime} \stackrel{c_{1}^{\prime}, c_{2}^{\prime}}{\longrightarrow} C^{\prime \prime}$, if $c_{1} \leq c_{2}$ and $c_{1}^{\prime} \leq c_{2}^{\prime}$, then $c_{1}^{\prime} \circ c_{1} \leq c_{2}^{\prime} \circ c_{2}$. A prefunctor $F: \mathscr{C} \rightarrow \mathscr{D}$ between two categorders consists of an assignment $F:$ ob $\mathscr{C} \rightarrow$ ob $\mathscr{D}$ and, for all $C, C^{\prime} \in \mathrm{ob} \mathscr{C}$, a function $F: \mathscr{C}\left(C, C^{\prime}\right) \rightarrow \mathscr{D}\left(F C, F C^{\prime}\right)$. The prefunctor $F: \mathscr{C} \rightarrow \mathscr{D}$ is said to be monotone if $F c \leq F c^{\prime}$ whenever $c \leq c^{\prime}$, for all $C \stackrel{c, c^{\prime}}{\longrightarrow} C^{\prime}$. Finally, a monotone prefunctor $F: \mathscr{C} \rightarrow \mathscr{D}$ is subfunctorial if the conditions

- $F\left(\operatorname{id}_{C}\right) \leq \operatorname{id}_{F C}$, for all $C \in \mathrm{ob} \mathscr{C}$

- $F\left(c^{\prime} \circ c\right) \leq F c^{\prime} \circ F c$ for all morphisms $C \stackrel{c}{\longrightarrow} C^{\prime} \stackrel{c^{\prime}}{\longrightarrow} C^{\prime \prime}$ in $\mathscr{C}$

hold. A subfunctorial monotone prefunctor will be called a functorder.

With the obvious notions of identity functorders and composition of functorders one obtains the category $\mathbf{C a t}_{\mathbf{1}}$ of all small categorders (where small means the underlying category is small) and functorders.

Remark 2.2. Notice that the notion of categorder is related to the notion of category enriched in the category Ord of ordered sets, but the notion of enriched functor is stronger than that of functorders, in particular an enriched functor is a functor between the underlying categories, while a functorder need not be.

We now describe the relationships depicted in

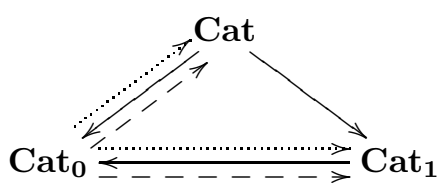

taken from the top level of the diagram above. The functors on the top left are adjoints, with left adjoint on top, but at the base of the diagram only the dashed arrow is a right adjoint. Each of the two triangles with either two dashed sides or two dotted sides commutes, as does the triangle of solid arrows. In more detail, the two functors leading from right to left are the forgetful functors which forget the morphisms and only remember the set of objects. The functor Cat $\rightarrow \mathbf{C a t}_{\mathbf{1}}$ maps a category $\mathscr{C}$ to the categorder having $\mathscr{C}$ as underlying category, endowed with the 
reflexive ordering on each hom set. Noting that every functor between categories is automatically a functorder between the associated categorders we may identify Cat as a full subcategory of Cat ${ }_{1}$. More generally, while a functorder need not have an underlying functor, any functorder $F: \mathscr{C} \rightarrow \mathscr{D}$ to a category $\mathscr{D}$ is a functor between the underlying categories. The adjoints of the forgetful functors are given by the discrete and indiscrete categories associated to a set. Again, the obvious discrete categorder construction $\mathbf{C a t}_{\mathbf{0}} \rightarrow \mathbf{C a t}_{\mathbf{1}}$ constitutes a functor, but it is no longer left adjoint to the forgetful functor.

\section{THE POWER CATEGORDER OF A CATEGORDER}

The suitable extension of the power set construction to categories is the aim of this section. Of course, the concept we develop is the one achieving the goal of this work. The possibility of defining the power category of a category to consist of all subcategories of the given category is explored in [14.

Firstly, we extend the power set construction to operate on ordered sets instead of just sets. A subset $A \subseteq S$ is down closed if $y \in A$ and $x \leq y$ imply $x \in A$, for all $x, y \in S$. Then $\mathcal{P}_{0} S$, for an ordered set $S$ is the collection of all down closed subsets $A \subseteq S$. $\mathcal{P}_{0} S$ is endowed with the ordering given by set inclusion. We note that $s \mapsto \downarrow s=\{t \in S \mid t \leq s\}$ is an order embedding $S \rightarrow \mathcal{P}_{0} S$. In the special case where the ordering on $S$ is the reflexive relation, we note that $\mathcal{P}_{0}(S)$ is just the power set of $S$ and the embedding $S \mapsto \mathcal{P}_{0} S$ is the inclusion by singletons $s \mapsto\{s\}$. More generally, for any subset $A \subseteq S$, let $\downarrow A=\bigcup_{a \in A}(\downarrow a)$, called the down closure of $A$.

Let us now fix a small categorder $\mathscr{C}$, for which we define a categorder $\mathcal{P}_{1} \mathscr{C}$. Let $\operatorname{ob}\left(\mathcal{P}_{1} \mathscr{C}\right)=\mathcal{P}_{0}(\mathrm{ob}(\mathscr{C}))$, namely all collections $\mathcal{C}$ of objects of $\mathscr{C}$. Since hom sets are pair-wise disjoint, the orderings on each $\mathscr{C}\left(C, C^{\prime}\right)$ induce an ordering on mor $\mathscr{C}$. The following down closures are computed in this ambient ordered set. For objects $\mathcal{C}$ and $\mathcal{C}^{\prime}$ in $\operatorname{ob}\left(\mathcal{P}_{1} \mathscr{C}\right)$ let $\operatorname{id}_{\mathcal{C}}=\downarrow\left\{\operatorname{id}_{C} \mid C \in \mathcal{C}\right\}$ and let $\mathscr{C}\left(\mathcal{C}, \mathcal{C}^{\prime}\right)=\bigcup_{C \in \mathcal{C}, C^{\prime} \in \mathcal{C}^{\prime}} \mathscr{C}\left(C, C^{\prime}\right)$. The morphisms in $\mathcal{P}_{1} \mathscr{C}$ are then given by $\left(\mathcal{P}_{1} \mathscr{C}\right)\left(\mathcal{C}, \mathcal{C}^{\prime}\right)=\mathcal{P}_{0}\left(\mathscr{C}\left(\mathcal{C}, \mathcal{C}^{\prime}\right)\right)$, where we use the $\mathcal{P}_{0}$ construction on ordered sets. In other words, a morphism in $\left(\mathcal{P}_{1} \mathscr{C}\right)\left(\mathcal{C}, \mathcal{C}^{\prime}\right)$ is a down closed collection $\mathbf{c}$ of morphisms in $\mathscr{C}$ with appropriate domains and codomains. The composition of $\mathcal{C} \stackrel{\mathbf{c}}{\longrightarrow} \mathcal{C}^{\prime} \stackrel{\mathbf{c}^{\prime}}{\longrightarrow} \mathcal{C}^{\prime \prime}$ is $\mathbf{c}^{\prime} \circ \mathbf{c}=\downarrow\left\{c^{\prime} \circ c \mid c \in \mathbf{c}, c^{\prime} \in\right.$ $\left.\mathbf{c}^{\prime}\right\}$, i.e., the down closure of the set of all morphisms obtained as a composition of morphisms $c \in \mathbf{c}$ and $c^{\prime} \in \mathbf{c}^{\prime}$ (whenever the composition exists).

Remark 3.1. It is important to note that mor $\mathcal{P}_{1} \mathscr{C}$ is not just $\mathcal{P}_{0}(\operatorname{mor} \mathscr{C})$. For instance, the empty set of morphisms is a morphism in $\left(\mathcal{P}_{1} \mathscr{C}\right)\left(\mathcal{C}, \mathcal{C}^{\prime}\right)$ for all objects $\mathcal{C}, \mathcal{C}^{\prime} \in \operatorname{ob}\left(\mathcal{P}_{1} \mathscr{C}\right)$. Many other sets of morphisms appear as a single morphism in different hom sets in $\mathcal{P}_{1} \mathscr{C}$, so, of course, we silently mark them differently so as to obtain a category. Consequently, $\operatorname{mor}\left(\mathcal{P}_{1} \mathscr{C}\right)$ is significantly larger than $\mathcal{P}_{0}($ mor $\mathscr{C})$.

The following result justifies calling $\mathcal{P}_{1} \mathscr{C}$ the power categorder of $\mathscr{C}$.

Theorem 3.2. $\mathcal{P}_{1} \mathscr{C}$ is a categorder.

Proof. The verification is straightforward. We only address the arguments that make use of the monotonicity in each variable of the composition in a categorder (a property that follows at once from the definition), starting with the associativity of composition in $\mathcal{P}_{1} \mathscr{C}$. Consider $\mathcal{C} \stackrel{\mathbf{c}}{\rightarrow} \mathcal{C}^{\prime} \stackrel{\mathbf{c}^{\prime}}{\rightarrow} \mathcal{C}^{\prime \prime} \stackrel{\mathbf{c}^{\prime \prime}}{\rightarrow} \mathcal{C}^{\prime \prime \prime}$, for which we show that $\left(\mathbf{c}^{\prime \prime} \circ \mathbf{c}^{\prime}\right) \circ \mathbf{c}=\mathbf{c}^{\prime \prime} \circ \mathbf{c}^{\prime} \circ \mathbf{c}=\mathbf{c}^{\prime \prime} \circ\left(\mathbf{c}^{\prime} \circ \mathbf{c}\right)$, where we introduce the ternary composition 
$\mathbf{c}^{\prime \prime} \circ \mathbf{c}^{\prime} \circ \mathbf{c}=\downarrow\left\{c^{\prime \prime} \circ c^{\prime} \circ c \mid c \in \mathbf{c}, c^{\prime} \in \mathbf{c}^{\prime}, c^{\prime \prime} \in \mathbf{c}^{\prime \prime}\right\}$. Let $\psi \in\left(\mathbf{c}^{\prime \prime} \circ \mathbf{c}^{\prime}\right) \circ \mathbf{c}$, i.e., $\psi \leq \varphi \circ c$, where $\varphi \in \mathbf{c}^{\prime \prime} \circ \mathbf{c}^{\prime}$ and $c \in \mathbf{c}$. Thus, $\varphi \leq c^{\prime \prime} \circ c^{\prime}$, for some $c^{\prime} \in \mathbf{c}^{\prime}$ and $c^{\prime \prime} \in \mathbf{c}^{\prime \prime}$. It now follows that $\psi \leq \varphi \circ c \leq\left(c^{\prime \prime} \circ c^{\prime}\right) \circ c=c^{\prime \prime} \circ c^{\prime} \circ c$, and thus $\psi \in \mathbf{c}^{\prime \prime} \circ \mathbf{c}^{\prime} \circ \mathbf{c}$. We thus conclude that $\left(\mathbf{c}^{\prime \prime} \circ \mathbf{c}^{\prime}\right) \circ \mathbf{c} \subseteq \mathbf{c}^{\prime \prime} \circ \mathbf{c}^{\prime} \circ \mathbf{c}$. The reverse inclusion is immediate, so equality follows, and the second claimed equality is obtained similarly. The neutrality of the identities $i_{\mathcal{C}}$ is a similar argument.

Notice that $\operatorname{id}_{\{C\}}=\downarrow\left\{\operatorname{id}_{C}\right\}$ and that $\mathscr{C}\left(\{C\},\left\{C^{\prime}\right\}\right)=\mathscr{C}\left(C, C^{\prime}\right)$, and thus $\left(\mathcal{P}_{1} \mathscr{C}\right)\left(\{C\},\left\{C^{\prime}\right\}\right)=\mathcal{P}_{0}\left(\mathscr{C}\left(C, C^{\prime}\right)\right)$ (again, we mean the order theoretic version of $\left.\mathcal{P}_{0}\right)$, for all $C, C^{\prime} \in \mathrm{ob}(\mathscr{C})$. Consequently, the assignment $C \mapsto\{C\}$ and

$C \stackrel{c}{\rightarrow} C^{\prime} \mapsto\{C\} \stackrel{\downarrow\{c\}}{\longrightarrow}\left\{C^{\prime}\right\}$ is defined and constitutes a functorder $\eta_{\mathscr{C}}: \mathscr{C} \rightarrow \mathcal{P}_{1}(\mathscr{C})$, essentially the inclusion by singletons. Of particular importance to the proof of Theorem 6.1 below, we note that when $\mathscr{C}$ is a category, i.e., a categorder with the reflexive order on each hom set, we have that $\left(\mathcal{P}_{1} \mathscr{C}\right)\left(\mathcal{C}, \mathcal{C}^{\prime}\right)=\mathcal{P}_{0}\left(\mathscr{C}\left(\mathcal{C}, \mathcal{C}^{\prime}\right)\right)$, the ordinary power set ordered by inclusion.

We note that $\mathcal{P}_{1}$ generally does not preserve discrete categories. In fact, the only discrete category $\mathscr{D}$ such that $\mathcal{P}_{1} \mathscr{D}$ is itself discrete is $\mathscr{D}=\emptyset$, the initial category. The precise way in which $\mathcal{P}_{1}$ extends $\mathcal{P}_{0}$ along the discrete category construction $\Delta:$ Cat $_{\mathbf{0}} \rightarrow \mathbf{C a t}_{\mathbf{1}}$ is detailed in the following result.

Lemma 3.3. For every set $S$ there exists a faithful functorder $\iota_{S}:\left(\Delta \circ \mathcal{P}_{0}\right) S \rightarrow$ $\left(\mathcal{P}_{1} \circ \Delta\right) S$ which is the identity on objects. In particular, $\mathrm{ob}\left(\mathcal{P}_{1} \Delta S\right)=\mathcal{P}_{0} S$.

Proof. The fact that ob $\left(\mathcal{P}_{1} \Delta S\right)=\mathcal{P}_{0} S$ is trivial. Extending the identity function to a functorder $\Delta \mathcal{P}_{0} S \rightarrow \mathcal{P}_{1} \Delta S$ is trivially obtained by inclusion of singletons.

\section{The DiReCt IMAGE FUnCTORDER}

With the power categorder constructed we now address the functoriality of $\mathcal{P}_{1}$. We first construct, for a given functorder $F: \mathscr{C} \rightarrow \mathscr{D}$, its associated direct image functorder $F_{\rightarrow}: \mathcal{P}_{1} \mathscr{C} \rightarrow \mathcal{P}_{1} \mathscr{D}$, which is given by $F_{\rightarrow}(\mathcal{C})=\{F C \mid C \in \mathcal{C}\}$, for all $\mathcal{C} \in \mathrm{ob}\left(\mathcal{P}_{1} \mathscr{C}\right)$, and $F_{\rightarrow}(\mathbf{c})=\downarrow\{F c \mid c \in \mathbf{c}\}$, for all morphisms $\mathbf{c}$ of $\mathcal{P}_{1} \mathscr{C}$.

Theorem 4.1. $F_{\rightarrow}: \mathcal{P}_{1} \mathscr{C} \rightarrow \mathcal{P}_{1} \mathscr{D}$ is a functorder.

Proof. The verification is straightforward. Let us for instance verify that $F_{\rightarrow}\left(\operatorname{id}_{\mathcal{C}}\right) \leq$ $\operatorname{id}_{F_{\rightarrow} \mathcal{C}}$, where $\mathcal{C} \in \mathrm{ob} \mathcal{P}_{1} \mathscr{C}$, remembering that $\leq$ is given by set inclusion. Indeed, $F_{\rightarrow}\left(\operatorname{id}_{\mathcal{C}}\right)=\downarrow\left(F\left(\downarrow\left(\left\{\operatorname{id}_{C} \mid C \in \mathcal{C}\right\}\right)\right)\right)$ while $\operatorname{id}_{F \rightarrow \mathcal{C}}=\downarrow\left\{\operatorname{id}_{F C} \mid C \in \mathcal{C}\right\}$. Thus, if $d \in F_{\rightarrow}\left(\operatorname{id}_{\mathcal{C}}\right)$, then $d \leq F c$, where $c \in \operatorname{mor} \mathscr{C}$ with $c \leq \operatorname{id}_{C}$ for some $C \in \mathcal{C}$. It then follows that $d \leq F c \leq F\left(\operatorname{id}_{C}\right) \leq \operatorname{id}_{F C}$, and thus $d \in \operatorname{id}_{F_{\rightarrow} \mathcal{C}}$, as needed.

Remark 4.2. Note that $F_{\rightarrow}$ need not be a functor, even if $F$ is. This can be seen by computing $F_{\rightarrow}$ for the functor $K$ described in Section 1 .

Theorem 4.3. Defining $\mathcal{P}_{1}(F)=F_{\rightarrow}$ turns $\mathcal{P}_{1}$ into a covariant endofunctor on Cat $_{1}$.

Proof. Again, a straightforward verification shows that indeed $\left(\mathrm{id}_{\mathscr{C}}\right)_{\rightarrow}=\mathrm{id}_{\mathscr{C}}$ and $(G \circ F)_{\rightarrow}=G_{\rightarrow} \circ F_{\rightarrow}$ hold true for all categorders $\mathscr{C}$ and functorders $\mathscr{C} \stackrel{G}{\rightarrow} \mathscr{D} \stackrel{F}{\rightarrow}$ $\mathscr{E}$.

Remark 4.2 can be restated now as the fact that $\mathcal{P}_{1}$ : $\mathbf{C a t}_{\mathbf{1}} \rightarrow \mathbf{C a t}_{\mathbf{1}}$ does not restrict to an endofunctor on the subcategory Cat of categories and functors. This is precisely the reason why Cat was extended to $\mathbf{C a t}_{\mathbf{1}}$. 
The relationship between the functors $\mathcal{P}_{0}$ and $\mathcal{P}_{1}$ along the discrete categorder functor $\Delta: \mathbf{C a t}_{\mathbf{0}} \rightarrow \mathbf{C a t}_{\mathbf{1}}$ and the forgetful functor $G: \mathbf{C a t}_{\mathbf{1}} \rightarrow \mathbf{C a t}_{\mathbf{0}}$ is summarized next. The trivial proof is omitted

Lemma 4.4. The functorders constructed in Lemma 3.3 form the components of a natural transformation $\iota: \Delta \circ \mathcal{P}_{0} \rightarrow \mathcal{P}_{1} \circ \Delta$. Further, $\mathcal{P}_{0}=G \circ \mathcal{P}_{1} \circ \Delta$.

\section{THE POWER CATEGORDER MONAD}

In this section we present the monad structure on the power categorder functor $\mathcal{P}_{1}$. Recall that the Manes monad structure on $\mathcal{P}_{0}$ : $\mathbf{C a t}_{\mathbf{0}} \rightarrow \mathbf{C a t}_{\mathbf{0}}$ is given by the unit $\eta: \mathrm{id}_{\text {Cat }_{0}} \rightarrow \mathcal{P}_{0}$ with components $\eta_{S}: S \rightarrow \mathcal{P}_{0} S$ with $s \mapsto\{s\}$, and multiplication $\mu: \mathcal{P}_{0}^{2} \rightarrow \mathcal{P}_{0}$ given by the components $\mu_{S}: \mathcal{P}_{0}^{2} S \rightarrow \mathcal{P}_{0} S$ by taking unions. It is not hard to see that our extension above of $\mathcal{P}_{0}$ to a functor Ord $\rightarrow$ Ord on the category of ordered sets and order preserving functions also gives rise to a monad, where the unit $\eta$ : id ${ }_{\text {Ord }} \rightarrow \mathcal{P}_{0}$ is given by $\eta_{S}(s)=\downarrow\{s\}$, and $\mu_{S}$ is still given by unions.

In Section 3 we already obtained the components $\eta_{\mathscr{C}}: \mathscr{C} \rightarrow \mathcal{P}_{1} \mathscr{C}$, given by $C \mapsto$ $\{C\}$ and $c \mapsto \downarrow\{c\}$, and it is easy to verify that they form a natural transformation id $_{\text {Cat }_{1}} \rightarrow \mathcal{P}_{1}$. We shall now obtain a multiplication natural transformation $\mu: \mathcal{P}_{1}^{2} \rightarrow$ $\mathcal{P}_{1}$. Recall that we extended the notation $\mathscr{C}\left(C, C^{\prime}\right)$ to collection $\mathcal{C}, \mathcal{C}^{\prime}$ of objects by means of $\mathscr{C}\left(\mathcal{C}, \mathcal{C}^{\prime}\right)=\bigcup_{C \in \mathcal{C}, C^{\prime} \in \mathcal{C}^{\prime}} \mathscr{C}\left(C, C^{\prime}\right)$. We may further extend the notation to families $\mathbb{C}, \mathbb{C}^{\prime}$ of collections of objects by defining $\mathscr{C}\left(\mathbb{C}, \mathbb{C}^{\prime}\right)=\bigcup_{\mathcal{C} \in \mathbb{C}, \mathcal{C}^{\prime} \in \mathbb{C}^{\prime}} \mathscr{C}\left(\mathcal{C}, \mathcal{C}^{\prime}\right)$.

Looking into the structure of $\mathcal{P}_{1}^{2} \mathscr{C}$ we have ob $\left(\mathcal{P}_{1}^{2} \mathscr{C}\right)=\mathcal{P}_{0}^{2}(\mathrm{ob} \mathscr{C})$ and $\left(\mathcal{P}_{1}^{2} \mathscr{C}\right)\left(\mathbb{C}, \mathbb{C}^{\prime}\right)=$ $\mathcal{P}_{0}\left(\left(\mathcal{P}_{1} \mathscr{C}\right)\left(\mathbb{C}, \mathbb{C}^{\prime}\right)\right)=\mathcal{P}_{0}^{2}\left(\mathscr{C}\left(\mathbb{C}, \mathbb{C}^{\prime}\right)\right)$. Thus, given a categorder $\mathscr{C}$, we define the following functorder $\mu_{\mathscr{C}}: \mathcal{P}_{1}^{2} \mathscr{C} \rightarrow \mathcal{P}_{1} \mathscr{C}$. On objects $\mu_{\mathscr{C}}: \mathcal{P}_{0}\left(\mathcal{P}_{0}(\right.$ ob $\left.\mathscr{C})\right) \rightarrow \mathcal{P}_{0}($ ob $\mathscr{C})$ is given by $\mu_{\mathrm{ob}(\mathscr{C})}$, the component of the Manes multiplication for $\mathcal{P}_{0}$. On morphisms, $\mu_{\mathscr{C}}:\left(\mathcal{P}_{1}^{2} \mathscr{C}\right)\left(\mathbb{C}, \mathbb{C}^{\prime}\right) \rightarrow\left(\mathcal{P}_{1} \mathscr{C}\right)\left(\mu_{\mathscr{C}} \mathbb{C}, \mu_{\mathscr{C}} \mathbb{C}^{\prime}\right)$ is the function $\mathcal{P}_{0}^{2}\left(\mathscr{C}\left(\mathbb{C}, \mathbb{C}^{\prime}\right)\right) \rightarrow$ $\mathcal{P}_{0}\left(\mathscr{C}\left(\mu_{\mathscr{C}} \mathbb{C}, \mu_{\mathscr{C}} \mathbb{C}^{\prime}\right)\right)$ given by $\mu_{\mathscr{C}}\left(\mathbb{C}, \mathbb{C}^{\prime}\right)$, the component of the multiplication for $\mathcal{P}_{0}$ as a monad on Ord, noting indeed that $\mathscr{C}\left(\mathbb{C}, \mathbb{C}^{\prime}\right)=\mathscr{C}\left(\mu_{\mathscr{C}} \mathbb{C}, \mu_{\mathscr{C}} \mathbb{C}^{\prime}\right)$.

Theorem 5.1. The functor $\mathcal{P}_{1}: \mathbf{C a t}_{1} \rightarrow \mathbf{C a t}_{1}$ together with the natural transformations $\eta: \operatorname{id}_{\mathbf{C a t}_{1}} \rightarrow \mathcal{P}_{1}$ and $\mu: \mathcal{P}_{1}^{2} \rightarrow \mathcal{P}_{1}$ form a monad.

Proof. This is once more a rather straightforward task, very similar to the proof that the Manes monad $\mathcal{P}_{0}$ : Set $\rightarrow$ Set is a monad.

The power categorder monad $\mathcal{P}_{1}$ is related to the power set monad $\mathcal{P}_{0}$ in the following manner, which is the formal presentation of the claim that the monad $\mathcal{P}_{0}$ : Set $\rightarrow$ Set is the object part of $\mathcal{P}_{1}$. Let us recall the notion of a functor of monads (see [16]). Given a monad $(S, \mu, \eta)$ on a category $\mathbf{C}$ and a monad $(T, \mu, \eta)$ on a category $\mathbf{D}$, a functor of monads is a functor $F: \mathbf{D} \rightarrow \mathbf{C}$ together with a natural transformation $\sigma: S \circ F \rightarrow F \circ T$ satisfying $F \eta=\sigma \circ \eta F$ and $\sigma \circ \mu F=F \mu \circ \sigma T \circ S \sigma$. It is trivial to verify that the forgetful functor $G$ : $\mathbf{C a t}_{\mathbf{1}} \rightarrow \mathbf{C a t}_{\mathbf{0}}$ is a monad functor when considered with the natural transformation $\sigma: \mathcal{P}_{0} \circ G \rightarrow G \circ \mathcal{P}_{1}$, which in fact is a natural isomorphism. In contrast, the discrete categorder functor $\Delta: \mathbf{C a t}_{\mathbf{0}} \rightarrow$ Cat $_{1}$ together with the natural transformation $\iota$ (see Lemma 3.3 and Lemma 4.4) fail to form a monad functor, since $\iota$ relates the natural transformations in the wrong order. In other words, the monad $\mathcal{P}_{0}$ : Set $\rightarrow$ Set does not embed in $\mathcal{P}_{1}$. 


\section{PARTIAL MULTIVALUED FUNCTORS}

In this section we obtain $\mathbf{C a t}_{\mathbf{1}}$ as a framework for partial multivalued functors, analogously to how Set serves as a framework for partial multivalued functions.

Let $\mathbf{R e l}_{\mathbf{1}}$ be the Kleisli category of the monad $\mathcal{P}_{1}$ : $\mathbf{C a t}_{\mathbf{1}} \rightarrow \mathbf{C a t}_{\mathbf{1}}$. Thus, the objects of $\mathbf{R e l}_{\mathbf{1}}$ are all small categorders, and morphisms $F: \mathscr{C} \rightarrow \mathscr{D}$ are given by morphisms $\hat{F}: \mathscr{C} \rightarrow \mathcal{P}_{1} \mathscr{D}$ in $\mathbf{C a t}_{1}$. The identity $\operatorname{id}_{\mathscr{C}}: \mathscr{C} \rightarrow \mathscr{C}$ at $\mathscr{C}$ is the component $\eta_{\mathscr{C}}: \mathscr{C} \rightarrow \mathcal{P}_{1} \mathscr{C}$ of the natural transformation from Section 5 , and the composition of $F: \mathscr{C} \rightarrow \mathscr{D}$ with $G: \mathscr{D} \rightarrow \mathscr{E}$ is given by $\mathscr{C} \stackrel{\hat{F}}{\rightarrow} \mathcal{P}_{1} \mathscr{D} \stackrel{\mathcal{P}_{1} G}{\longrightarrow} \mathcal{P}_{1} \mathcal{P}_{1} \mathscr{E} \stackrel{\mu_{\mathscr{E}}}{\longrightarrow} \mathcal{P}_{1} \mathscr{E}$, utilizing the multiplication natural transformation $\mu$ defined in Section 5 . The following result is the main theorem of this work where the category of categories and partial multivalued functors is identified as a full subcategory of $\mathbf{R e l}_{\mathbf{1}}$.

Theorem 6.1. The category $\mathbf{C a t}_{\mathbf{p m v}}$ is isomorphic to the full subcategory of $\mathbf{R e l}_{\mathbf{1}}$ spanned by the categories.

Proof. The claimed isomorphism is the identity on objects, and maps a morphism $F: \mathscr{C} \rightarrow \mathscr{D}$ between two categories in $\operatorname{Rel}_{\mathbf{1}}$, i.e., a functorder $\hat{F}: \mathscr{C} \rightarrow \mathcal{P}_{1} \mathscr{D}$, to the following partial multivalued functor $G: \mathscr{C} \rightarrow \mathscr{D}$. Let us write $F_{0}$ and $F_{1}$ for the object and morphism parts, respectively, of the functorder $\hat{F}$. Thus, $F_{0}: \mathrm{ob} \mathscr{C} \rightarrow \mathcal{P}_{0}(\mathrm{ob} \mathscr{D})$ is a function, giving rise to a partial multivalued function $G_{0}: \mathrm{ob} \mathscr{C} \rightarrow \mathrm{ob} \mathscr{D}$. The morphism part of $\hat{F}$ consists of components $F_{1}: \mathscr{C}\left(C, C^{\prime}\right) \rightarrow$ $\mathcal{P}_{0}\left(\mathscr{D}\left(F_{0} C, F_{0} C^{\prime}\right)\right)$, giving rise to a partial multivalued function $G_{1}: \mathscr{C}\left(C, C^{\prime}\right) \rightarrow$ $\mathscr{D}\left(G_{0} C, G_{0} C^{\prime}\right)$. The condition that if $D \stackrel{d}{\rightarrow} D^{\prime} \in G_{1}\left(C \stackrel{c}{\rightarrow} C^{\prime}\right)$, then $D \in G_{0} C$ and $D^{\prime} \in G_{0} C^{\prime}$ follows at once. Further, since $\hat{F}\left(\operatorname{id}_{C}\right) \leq \operatorname{id}_{F C}$ translates to $\hat{F}\left(\operatorname{id}_{C}\right) \subseteq$ $\left\{\operatorname{id}_{D} \mid D \in F C\right\}$, it follows that if $D \stackrel{d}{\rightarrow} D^{\prime} \in G_{1}\left(\operatorname{id}_{C}\right)$, then $D=D^{\prime}$ and $d=\operatorname{id}_{D}$. Finally, from $\hat{F}\left(c^{\prime} \circ c\right) \leq \hat{F} c^{\prime} \circ \hat{F} c$, i.e., $\hat{F}\left(c^{\prime} \circ c\right) \subseteq \hat{F} c^{\prime} \circ \hat{F} c$, it follows that if $d^{\prime \prime} \in G_{1}\left(c^{\prime \prime}\right)$, and $c^{\prime \prime}=c^{\prime} \circ c$, i.e., $d \in \hat{F}\left(c^{\prime} \circ c\right)$, then $d \in \hat{F} c^{\prime} \circ \hat{F} c$, implying the existence of $d \in G_{1} c$ and $d^{\prime} \in G_{1} c^{\prime}$ with $d^{\prime \prime}=d^{\prime} \circ d$. The verification that the composition in $\mathbf{R e l}_{\mathbf{1}}$ agrees with the composition in $\mathbf{C a t}_{\mathbf{p m v}}$ and that identities agree, is easy. Finally, constructing a morphism in $\mathbf{R e l}_{\mathbf{1}}$ from a given partial multivalued functor is similar.

Referring to the middle layer of the main diagram from section \$1, we first mention that the same commutativity relations hold as for the top layer (and these were described in section \$2). The functors themselves are the obvious ones; the functors going from right to left are the forgetful functors (forgetting morphisms) and the functors emanating from $\mathbf{R e l}_{\mathbf{0}}$ are the immediate versions of the discrete and indiscrete constructions. However, due to a phenomenon related to the failure of $\mathcal{P}_{1}$ to preserve discreteness, these functors are no longer left, respectively right, adjoint to the forgetful functors (as is easily verified). We note (cf. the closing discussion in section (55) that $\mathbf{R e l}_{\mathbf{1}} \rightarrow \mathbf{R e l}_{\mathbf{0}}$ is the functor induced by the fact that $\mathcal{P}_{0}$ is the object part of $\mathcal{P}_{1}$, while neither of the functors in the other direction is induced by an immediate structural relationship between the two monads. Finally, the functors leading from the top layer to the middle layer all view a single-valued entity (i.e., function, functor, or functorder) as a particular kind of a partial multivalued entity in the corresponding category in the middle layer.

Recall ([13]) that the Eilenberg-Moore category of $\mathcal{P}_{0}$ is the category CSLat of $_{0}$ complete join semi lattices and join preserving homomorphisms. Considering the 
Eilenberg-Moore category of $\mathcal{P}_{1}$ leads to the third and final layer of the diagram. To be precise, CSLat $\boldsymbol{1}_{1}$ is the Eilenberg-Moore category of $\mathcal{P}_{1}$, and Cat CSLat $_{\text {is }}$ its full subcategory spanned by the categories. An object in CSLat $\mathbf{C}_{\mathbf{1}}$ is thus a categorder $\mathscr{C}$ together with a structure functorder $a: \mathcal{P}_{1} \mathscr{C} \rightarrow \mathscr{C}$, satisfying a unit and associativity rules. In some more detail, the structure functorder $a$ consists of a function $a: \mathcal{P}_{0}(\operatorname{ob} \mathscr{C}) \rightarrow \operatorname{ob} \mathscr{C}$ and, for all $\mathcal{C}, \mathcal{C} \subseteq$ ob $\mathscr{C}$, a function $\mathcal{P}_{0}\left(\mathscr{C}\left(\mathcal{C}, \mathcal{C}^{\prime}\right)\right) \rightarrow$ $\mathscr{C}\left(a(\mathcal{C}), a\left(\mathcal{C}^{\prime}\right)\right)$. Note that the objects of $\mathbf{C a t}_{\text {CSLat }}$ are defined purely categorically, rather than categorderly, since a functorder $\mathcal{P}_{1} \mathscr{C} \rightarrow \mathscr{C}$ for a category $\mathscr{C}$ is just a functor of the underlying categories.

The relationship between the monads $\mathcal{P}_{0}$ and $\mathcal{P}_{1}$ implies that every $\mathcal{P}_{1}$-algebra gives rise to a $\mathcal{P}_{0}$-algebra, namely forgetting the morphisms of an object $\mathscr{C}$ in CSLat $_{1}$ results in a complete join semi lattice. Moreover, (and here Remark 3.1 is of importance), each hom set $\mathscr{C}\left(C, C^{\prime}\right)$ carries the structure of a complete join semi lattice. Cat CSLat is thus clearly related to the category of categories internal to CSLat, though there is much more to $\mathscr{C}$ than just the existence of complete join semi lattice structures on the objects and on the hom sets. Fully investigating these structures will take us too far afield though, and so, other than noting that the functors leading from layer two to layer three are the usual embeddings of the Kleisli category in the Eilenberg-Moore category, we conclude by discussing the omittance of a discrete and indiscrete constructions at the bottom layer. For instance, given a complete join semi lattice $S$ with three elements $u, v, w$ with $u \vee w \neq v \vee w$, the discrete category $\Delta S$ does not carry the structure of an object

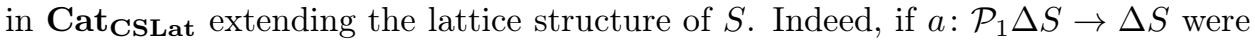
such a structure, then noting than $\left\{\operatorname{id}_{w}\right\}:\{u, w\} \rightarrow\{v, w\}$ is a morphism in $\mathcal{P}_{1} \Delta S$, one must then have $a\left(\left\{\operatorname{id}_{w}\right\}\right): u \vee w \rightarrow v \vee w$ in $\Delta S$, but no such morphism exists.

\section{REFERENCES}

[1] David J. Anick. Connections between Yoneda and Pontrjagin algebras. In Algebraic topology, Aarhus 1982 (Aarhus, 1982), volume 1051 of Lecture Notes in Math., pages 331-350. Springer, Berlin, 1984.

[2] IC Baianu, R Brown, and JF Glazebrook. Categorical ontology of complex spacetime structures: the emergence of life and human consciousness. Axiomathes, 17(3-4):223-352, 2007.

[3] Ivan Contreras. Relational symplectic groupoids and poisson sigma models with boundary (ph.d. dissertation). arXiv preprint arXiv:1306.3943, 2013.

[4] Ernst-Erich Doberkat. Semi-pullbacks and bisimulations in categories of stochastic relations. In Automata, Languages and Programming, pages 996-1007. Springer, 2003.

[5] Ernst-Erich Doberkat. Stochastic relations: Congruences, bisimulations and the hennessymilner theorem. SIAM Journal on Computing, 35(3):590-626, 2005.

[6] Ernst-Erich Doberkat. Eilenberg-moore algebras for stochastic relations. Information and Computation, 204(12):1756-1781, 2006.

[7] Ernst-Erich Doberkat. Kleisli morphisms and randomized congruences for the giry monad. Journal of Pure and Applied Algebra, 211(3):638-664, 2007.

[8] Kathryn Hess. Perturbation and transfer of generic algebraic structure. In Higher homotopy structures in topology and mathematical physics (Poughkeepsie, NY, 1996), volume 227 of Contemp. Math., pages 103-143. Amer. Math. Soc., Providence, RI, 1999.

[9] Kathryn P. Hess. Mild and tame homotopy theory. J. Pure Appl. Algebra, 84(3):277-310, 1993.

[10] Chris Heunen, Ivan Contreras, and Alberto S. Cattaneo. Relative Frobenius algebras are groupoids. J. Pure Appl. Algebra, 217(1):114-124, 2013.

[11] Sebastian Link. On the implication of multivalued dependencies in partial database relations. Internat. J. Found. Comput. Sci., 19(3):691-715, 2008. 
[12] Saunders Mac Lane. Categories for the working mathematician, volume 5 of Graduate Texts in Mathematics. Springer-Verlag, New York, second edition, 1998.

[13] Ernest Manes. A triple theoretic construction of compact algebras. In Sem. on Triples and Categorical Homology Theory (ETH, Zürich, 1966/67), pages 91-118. Springer, Berlin, 1969.

[14] Alfio Martini, Uwe Wolter, and E. Hermann Haeusler. Fibred and indexed categories for abstract model theory. Log. J. IGPL, 15(5-6):707-739, 2007.

[15] Alan L. Selman. A taxonomy of complexity classes of functions. J. Comput. System Sci., 48(2):357-381, 1994.

[16] Ross Street. The formal theory of monads. J. Pure Appl. Algebra, 2(2):149-168, 1972. 